Monatsschrift f. Geburtshülfe u. Gynäkologie 1925;70:115-119

\title{
V. Literaturverzeichnis
}

\section{Qeburtshilfe.}

Andersen, X-Strahlen bei px-omortaler Diagnose fetaler Monstrumbildung.

Amer. Journ. Obst. gyn. IX. 3. S. 382. Ballhorn, Zur Placent $3 / 8$-praevia-Therapie. Arch. f. Gyn.

Bd. 123. S. 646. Barna, Eine junge Eierstocksschwangerschaft. Z. f. G. Nr. 13. S. 718. Bauch, Zur Therapie des hohen Geradstandes. Z. f. G. Nr. 15. S. 821. Beloser, Die anatomische Begründung des Blasensprungs bei normalen

und vorzeitigen Geburten. Z. f. G. Nr. 12. S. 644. Bietmer, Zur Immunisierung Schwangerer und Wöchnerinnen gegen puerperale Streptokokkeninfektion. Z. f. G. Nr. 13. S. 674. Bland, Myoma uteri und Schwangerschaft, bes. betr. Tumornekrose.

Surg. gyn. obstet. XI. 3. S. 367. Brindeau und Lantuéjoul, Über den forceps Kjelland. Gynèc. obst.

XI. 3. S. 199. Browne, Abnormitäten der Nabelschnur als Ursache des antenatalen Absterbens. J. obst. gyn. Brit. Emp. Vol. 32. Nr. 1. S. 17. Bublitschenko, Zur Frage des Abortus spontaneus-praetematuralis. Z. f. G.

Nr. 15. S. 827. . Candia, G. de, Die Passage des schwefelsauren Chinin durch die Milch und sein Einfluß auf das Wohl des Neugeborenen. La clinica ostet.

XXVII. 4. S. 155. Clauser, Ein Fall von schwerer perniziöser aplastischer Schwangerschaftsanämie, behandelt mit kolloidalem Antimontrisulfat. Z. f. G. Nr. 14.

S. 752. Conrad, Über die Leitung der Nachgeburtsperiode. Munch, med. Woch.

Nr. 12. S. 481. Cosgrove und Waters, Eklampsie bei Uterus duplex und einer Niere.

Amer. J. obst. gyn. IX. 3. S. 360. Costa, Extraktion des Kopfes bei Steißlage bei lebenden Kind, vermittels

einer in den Mund eingeführten gestielten Platte. La clinica ostetr.

XXVII. 3. S. 97. Dipper, Zur Einschränkung der manuellen Untersuchung während der Geburt. Z. f. G. Nr. 16. S. 863. Eberhart, Geburtshilfliches Brevier (Fortsetzung). Med. Klin. Nr. 14. S.513 Feldmann, Zur Frage der ,Inneren Untersuchung durch die Hebamme”.

Munch, med. Woch. Nr. 12. S. 475. Fellner, Ein besonderes Phänomen bei beginnender Schwangerschaft.

Z. f. G. Nr. 16. S. 869. Framm, Primäre Rhinitis gonorrhoica bei einem Neugeborenen. Dtsch. med. Woch. Nr. 12. S. 472. Franquè, v., Abortbehandlung. Med. Klin. Nr. 12. S. 425. $8^{*}$

21 (j Líteraturverzeichnís.

Freid, Uber die Hänfigkeit der Dekapitation bei Querlagen, sowie über eine neue Methodik dieser Operation. Z. f. G. Nr. 16. S. 874. Frey, Walter, Das Herzgefäßsystem in der Gravidität. Klin. Woch.

Nr. 13. S. 577. Gaifami, Eignet sich die Kjellandzange für den praktischen Arzt ? La clinica ostet. XXVII. 4. S. 177. Ders., Einige Kontraindikationen der Hypophysenextrakte in Part $1 / 8 n$. 
La clinica ostet. XXVII. 3. S. 1 2. Geppert, Kritisches Sammelreferat über die Kíellandsche Zange. Dtscli.

med. Woch. Nr. 12. S. 499. Geßner, Zur diätetischen Prophylaxe der Eklampsie. Z. f. G. Nr. 14. S. 738. Halban-Seitz, Biologie und Pathologie des Weibes. Lief. 14 (S. 1-240):

O. Grosser, Entwicklungsgeschichte des Menschen von der Keimzelle bis zur Ausbildung der äußeren Körperform. Unter Berücksichtigung ihrer vergleichend-entwicklungsgeschichtlichen Grundlagen; vergleichende und menschliche Plazentationslehre. - Dietrich, Anatomie und Physiologie des $\bullet$ Fetus und Biologie der Plazenta. Heinsius, Der extraperitoneale vaginale kleine Kaiserschnitt. Z. f. G.

Nr. 14. S. 763. Henkel, Über die klinische Bewertung klimakterischer Blutungen. Dtsch. med. Woch. Nr. 13. S. 508. Heyn, Über kurzfristige Schwangerschaften. Arch, f. Gyn. Bd. 123. S. 534. Hofmeier, Beiträge zur forensischen Geburtshilfe. Munch, med. Woch.

Nr. 12. S. 479. Nr. 14. S. 566. Joachímowiíz, Studien über ein Salz des ß-Pyridons und einiger Nikotinsäurederivate in hirer Wirkung auf den Uterus. Arch. f. Gyn.

Bd. 123. S. 769. Kaíz, Über die Bedeutung der Austastung der Uterus post partum zur Entfernung zurückgebliebener Plazentarreste. Z. f. G. Nr. 12. S. 637. Kaufmann, Toxisches Exanthem bei einem Neugeborenen post partum.

Dtsch. med. Woch. Nr. 15. S. 608. Kelen, Über Anwendung der Kiellandschen Zange bei hochstehendem Steiß.

Z. f. G. Nr. 16. S. 871. Kiefer, Zur Wochenbettsmorbilität nach den intrauterinen Eingriffen in der Nachgeburtsperiode. Z. f. G. Nr. 16. S. 860. Kirstein und Schwieder, Über die essentielle puerperale Ischurie. Arch. f.

Gyn. Bd. 123. S. 503. Kleesattel, Über das Verhalten der Gallensäuren im Blut und Harn während

der Schwangerschaft. Arch. f. Gyn. Bd. 123. S. 638. Koch, Über einen Fall von spontaner zirkulärer Abstoßung der Portio vaginalis sub partu. Z. f. G. Nr. 16. S. 866. Kosmak, Subkutanes Emphysem nach der Geburtsarbeit. Surg. gyn.

obst, XL 3. S. 434. Krònig, Werner, Die rektale Untersuchung bei der Geburt und ihre Bedeutung für den praktischen Arzt. Arch. f. Gyn. Bd. 123. S. 542. Krupenikoff, Von den Bahnen der Verbreitung puerperaler Parametritis.

Z. f. G. Nr. 14. S. 765.

Literaturverzeichnis.

117

Küstner, Heinz, Pliysikalische und anatomische Untersuchungen der Bauchwand, speziell ihrer muskulösen Partien bei der Frau. Arch. f. Gyn. Bd. 123. S. 420. Leven, Beobachtungen an Zwillingen. Dtsch. med. Woch. Nr. 15. S. 609. Lichtenstein, Neue Prophylaxe in der Nachgeburtsperiode, einschließlich der Atonie und des Schüttelfrostes nach der Geburt. Munch, med. Woch. Nr. 15. S. 585. Litten, Über die Normalmaße des mit Weichteilen ausgekleideten weiblichen Beckens. Z. f. G. Nr. 15. S. 817. Lockhart, Bakteriologische Untersuchungen an Zervix und Vagina während der Schwangerschaft mit besonderer Beziehung zum Streptococcus 
haemolyticus. J. Obst. gyn. Brit. Emp. Vol. 32. Nr. 1. S. 46. Loiiros, N. C. und Fuß, Die Resistenz der Vaginalkeime als Gradmesser ihrer Virulenz. Klin. Woch. Nr. 15. S. 698. Meiner, Schwangerschaft nach Röntgenreizbestrahlung der Ovarien. Z. f.

G. Nr. 13. S. 682. גlerlelli, Kritik des klinischen Unterrichts. La clinica ostet. XXVII. Nr. 3. S. 116. Meyer, Robert, Uber einen Fall von doppelseitigem Ovotestis beim Neugeborenen, sowie über besondere Formen der Keimdrüsen-Geschwulstbilduhg bei Pseudohermaphroditismus und Hermaphroditismus verus sowie über gleichartige Geschwülste bei nichtzwittrigen Personen. Arch. f. Gyn. Bd. 123. S. 675. Momigliano, Über die Lipoide der Corpus luteum. Z. f. G. Nr. 13. S. 684. Ötíingen, von, Zum Kohlehydratstoffwechsel in der Plazenta. Z. f. G.

Nr. 12. S. 625. Pomini, Anaemia perniciosa graviditatis. La clinica ostet. XXVII. 3. S. 109. Randall, Das Gewicht bei Schwangerschaft. Am. J. obst. gyn. IX. 4. S.529. Reisí, Die Erweiterung des Unterrichts in der Rektaluntersuchung.

Z. f. G. Nr. 12. S. 629. Reveno und Welz, Die hämoklasische Krise. Probe in der Schwangerschaft. Am. J. obst. gyn. IX. 4. S. 535. Roller, Über die Erfahrungen mit Cuprokollargol ,Heyden”. Munch.

med. Woch. Nr. 13. S. 515. Rusch, Behandlung der Pyelitis graviditatis mit Verweilkatheter. Ein Fall. Surg. gyn. obst, XI. 3. S. 428. Saenger, Mein Schlußwort zur Kritik der Kiellandzange. Z. f. G. Nr. 14.

S. 762. Seynsche, Zur Verwendung des Glaskatheters unter der Geburt. Dtsch. med. Woch. Nr. 16. S. 660. Sharpe und Maclaire, Intrakraniale Hämorrhagie bei 400 aufeinander-

folgenden Neugeborenen. J. obst. gyn. Brit. Emp. Vol. 32. Nr. 1.S.79. Siegert, Versuche mit Somnifen in der Geburtshilfe. Z. f. G. Nr. 14. S. 748. Sommer, Schwangerschaft und Karzinom. Z. f. G. Nr. 14. S. 758. Sehmiü-Oíí, Die Geschlechtsbestimmung des Fötus in utero mit der serologischen Alkoholreaktion (Testesfremdreaktion) nach Lüttge-v. $\lambda$ lertz.

Dtsch. med. Woch. Nr. 12. S. 469. Slander, Eklampsiebehandlung nach Stroganoif. Amer. J.

obst. gyn.

IX. 3. S. 327.

118

Literaturverzeichøis.

Stork, Über die Achsendrehung des Uterus intra partum. Z. f. G. Nr. 12.

S. 641. Strachan, Physiologie der Plazenta. J. obst. gyn. Brit. Emp. Vol. 32.

Nr. 1. S. 89. Toth, Über die Bedeutung der Veränderung der kolloidalen Plasma struktur bei puerperalen septischen Erkrankungen. Arch. f. Ciyn.

Bd. 123. S. 623. Treuter, Eine bequeme Methode zur Prüfung von Hypophysenpräparaten.

Z. f. G. Nr. 15. S. 831. Vaudescal, Eigenartige Entwicklung einer Extra-uterin-Schwangerschaft. Spontangeburt ad terminum. Lebendes Kind. Tod durch Ver-

blutung in der Nachgeburtsperiode. La clinic a ostetr. XXVII. 4. S.184. Viana, Plazenta

undMilchabsonderung. La clinica ostetr. XXVII. 4. S.163. Vogt, Liquor cerebrospinalis rind

Blutzucker. Klin. Woch. Nr. 13. S. 597. Westphal, Ocenta, ein neues Lactagogon. Z. f. G. Nr. 13.

S. 719. Wielocli, Weiteres Material über die Eklampsie mit Berücksichtigung der

Hirndrucktheorie. Arch. f. Gyn. Bd. 123. S. 337. Wolfe, Fall von Cychlosoma, seltene fetale Mißbildung. Amer. J. obst.

gyn. IX. 4. S. 540. Wolf son, Blasendivertikel als Geburtshindernis. Ein Fall. Surg. gyn. 
obst. April 25. S. 524. Zimmermann, Vorteile und Nachteile der Kiellandschen Zange. Klin. Woch. Nr. 13. S. 600.

Gynäkologie.

Adler und Singer, Septische Infektion und Sepsistherapie. Med. Klin.

Nr. 12. S. 429. Albano, Endokrinisch-morphologische Studie in einem Fall von chronischem menstrualen Singultus. La clinica ostet. XXVII. 3. S. 99. Bisell, Kenale Papülome und ureterale Implantation. Chirurgische Be-

handlung beim Weibe. Ein Fall. Surg. gyn. obst. XL 3. S. 323. Blair Bell, Пendry, Armeít, Die spezifische Wirkung des Bleis auf das

C'horio-Epitheliom des Kaninchens, verglichen mit der von Kupfer,

Thallium und Thorium. J. obstet. gyn. Brit. Emp. Vol. 32. Nr. 1. S.1. Castaño, Behandlung der

Sterilität mit Diathermin. Gynéc. obst. XL 3.

- S. 207. Castellani, Taylor, Vaginale Mykosen. J. obst. gyn. Brit. Emp. Vol. 32.

Nr. 1. S. 69. Colby, Embryonale B, este der Harnblase. Surg. gyn. obst. April 25. S. 528. Coley, Klinische Beweise für äußerliche Lrsacheir der Krebsbiklung.

Surg. gyn. obst. XI. 3. S. 353. Conrad, Beitrag zur operativen Behandlung cles weiblichen

Genital-prolapses nach der Kiellandschen Methode. Z. f. G. Nr. 14. S. 743. de Courcy und

Maloney, Fibroma mesenterii. Ein Fall. Surg. gyn. obst.

XL 3. S. 402. Daniel, Tivberkulose des Corpus uteri. Gynéc. obst. XL 3. S. 161. Dickinson, Ein einfaches silbernes Punkt-Kauterium. Surg. gyn. obst.

April 25. S. 567. Douay, Die weibliche Sterilität. Gynéc. obstétr. XL 4. S. 283.

Tagesuachrichten und Personalien.

119

Dubreuil-Chambardel, Varianten des Verlaufes der Beckenarterien und der

unteren Extremitäten. Paris 1925. Masson \& Cie. Diihrßen, Beitrag zur Priorität und Technik

einiger berühmter gynäko-

logischer, vaginaler Operationen. Arch. f. Gyn. Bd. 123. S. 452. Eisner, Kritisches

Sammelreferat über die Serumbehandlung des inoperablen

Karzinoms. Dtsch. med. Woch. îsr. 15. S. 624. Fe,iner, Atresia Cervicis. Am. J. obst. gyn. IX. 4. S. 544. Fischer, Über psychogene Ursachen körperlicher Symptome in der Gynäko-

logie· und Geburtshilfe. Z. f. G. Nr. 15. S. 800. Fliegel und Slrauß, Zur „Arthtropathia ovaripriva" Menges. Z. f. G.

Nr. 12. S. 633. Flörken, Kosmetische Rüeksichten in der operativen Chirurgie des Halses, der Brust und des Bauches. Munch, med. Woch. Nr. 12. S. 463. Gänßle, Die

Wasserstoffionenkonzentration im Scheidensekret. Arch. f.

Gyn. Bd. 123. S. 602. Goto, Experimentelle Untersuchung der inneren Sekretion des Ovariums durch Rattenparabiose. Arch. f. Gyn. Bd. 123. S. 387. Gouverneur und Fabre, Die Zystoskopie bei Uteruskrebs vor und nach Be-

strahlung. 200 Fälle. Gynéc. obst. XI. 3. S. 189. Greil, Veto gegen die ovarielle temporäre

Sterilisierung. Z. f. G. Nr. 16.

S. 862. Halban-Seilz, Biolvgie und Pathologie des Weibes. Lief. 13 (Band V,

1. Hälfte): Nürnberger, Aktinomycosis, Echinokokkus, Bilharziosis. -

Weibel, Tuberkulose des weiblichen Genitalapparates. - G. A. Wagner,

Gonorrhöe cles weiblichen Geschlechtsapparates. Berlin-Wien 1925.

Urban \& Schwarzenberg. Helivig, Actinomycosis ovarii e tubae. Surg. gyn. obst. April 1925. S. 502. Herold, Zur Methodik der Blutkörperchensenkungsreaktion nach Henkel 
(Zentrifugensenkung in 5 Minuten). Z. f. G. Nr. 12. S. 634. Hurler, Beobacbtungen bei 400 Narzylenbetäubungen. Munch, med.

Woch. Nr. 12. S. 468.

Stralilentherapíe.

Döderlein, Gusiav, Gibt es eine primäre Mortalität bei der Radiumbehand-

lung des Uteruskarzinoms ? Z. f. G. Nr. 16. S. 852. Pordes, Die Strahlenbehandlung des g5mäkologischen Karzinoms (An-

merkung zur gleichnamigen Arbeit von Schollen und Vollz in Nr. 1).

Munch, med. Woch. Nr. 16. S. 653. Weber und Preiinger, Die Vereinheitlichung der

Röntgendosismessung.

Med. Klin. Nr. 15. S. 549.

Tagesnachrichten und Personalien.

Die L7niv.-Frauenklinik za Münster wurcle am 15. V. 25. gemeinsam mit den anderen klinischen Instituten durch eine würdige Feier ihrer hohen Aufgabe überliefert.

Die ursprünglich von $\mathrm{Ph}$. Jung durchgearbeiteten Plane sind später von A. Mayer nachhaltig geändert worden. Als Meister der Zisilierkunst für diese Anstalt hat sich Esch ein bleibendes Denkmal gesetzt. Die über- 